\title{
Survival after acute colon diverticulitis treated in hospital
}

\author{
Tom-Harald Edna • Aras Jamal Talabani • \\ Stian Lydersen • Birger Henning Endreseth
}

Accepted: 22 June 2014 / Published online: 3 July 2014

(C) The Author(s) 2014. This article is published with open access at Springerlink.com

\begin{abstract}
Purpose The aim of this study was to determine the short- and long-term relative survival as well as the causes of death in patients treated in hospital for acute colonic diverticulitis.

Materials and methods The study included all patients treated at Levanger Hospital for acute colonic diverticulitis between 1988 and 2012. Vital statistics were complete. The median observation time was 6.95 years (range $0.28-24.66$ ) or until death.

Results In total, 650 different patients were hospitalized with acute colonic diverticulitis. Among these patients, there were 851 admissions for the same disease during the 25 years. The admissions had the following diagnoses: simple diverticulitis, 738; abscess formation, 44; perforation and purulent peritonitis, 47; perforation and fecal peritonitis, 9; and intestinal obstruction, 13. During the observation time, 219 were dead
\end{abstract}

T.-H. Edna $(\bowtie) \cdot$ A. Jamal Talabani

Department of Surgery, Levanger Hospital, North-Trondelag

Hospital Trust, Levanger, Norway

e-mail: thedna@hnt.no

\section{T.-H. Edna}

Unit for Applied Clinical Research, Faculty of Medicine, Institute of Cancer Research and Molecular Medicine, Norwegian University of Science and Technology, Trondheim, Norway

\section{S. Lydersen}

Regional Centre for Child and Youth Mental Health and Child

Welfare - Central Norway, Faculty of Medicine, Norwegian

University of Science and Technology, Trondheim, Norway

\section{B. H. Endreseth}

Department of Gastroenterological Surgery, St Olavs Hospital, University of Trondheim, Trondheim, Norway

\section{B. H. Endreseth}

Institute of Cancer Research and Molecular Medicine, Faculty of Medicine, Norwegian University of Science and Technology, Trondheim, Norway and 431 were still alive. After the first admission, the 100 day relative survival in patients with uncomplicated diverticulitis was $97 \%$ (CI 95 to 99), with abscess formation $79 \%$ (62 to 89 ), with purulent peritonitis $84 \%$ (69 to 92 ), with fecal peritonitis $44 \%$ (10 to 74 ), and with intestinal obstruction $80 \%$ (38 to 96 ). After surviving the first 100 days, the estimated 5-year relative survival in the remaining 609 patients was $96 \%$ (CI 92 to 100) and 10-year survival was $91 \%$ (CI 84 to 97). In patients who survived the first 100 days, the different subtypes of diverticulitis yielded no significant differences in long-term relative survival. All patients who had been admitted with ASA score 4 were dead after 2 years.

Keywords Acute colonic diverticulitis · Short-term survival · Long-term survival

\section{Introduction}

Acute colonic diverticulitis is an increasingly common acute abdominal condition in the Western world. Most patients have uncomplicated acute diverticulitis, while 9-35\% develop serious acute complications due to colonic perforation, with abscess formation or diffuse peritonitis, or intestinal obstruction [1-5].

Inhospital mortality has been shown to occur in 0 to $17 \%$ of patients with abscess formation and 0.4 to $45 \%$ of patients with perforation and generalized peritonitis [6-11]. In series that also include patients with uncomplicated diverticulitis, the inhospital mortality rate is between 0.5 and $7 \%[6,7]$.

Population-based estimates of short- and long-term survival after admission for the different types of acute colonic diverticulitis are scarce. Patients with perforation or abscess had $15.5 \%$ 1-year mortality in a recent study from the UK [12]. Long-term mortality after different types of diverticulitis has been studied by Binda et al. [13] and after perforated 
diverticulitis by Vermeulen [14]. In those who survived the first episode with diverticulitis, long-term survival was not associated with the severity of the primary disease.

The objective of the present study was to assess short- and long-term relative survival in patients treated for the different subtypes of acute colonic diverticulitis in hospital. We also studied the causes of death in all patients.

\section{Patients and methods}

\section{Study population}

All patients hospitalized because of acute diverticulitis at Levanger Hospital, between January 1988 and December 2012, were identified through the patient administrative system. The records were reviewed and clinical data collected. A total of 650 patients were admitted 851 times: 519 were admitted once, 91 twice, 25 three times, 9 four times, 1 five times, 4 six times, and 1 ten times.

We classified the cases as uncomplicated or complicated acute diverticulitis. Acute complicated diverticulitis was further classified into four subcategories: abscess formation (Hinchey stage I or II), bowel obstruction related to stenosis, perforation with purulent peritonitis (Hinchey stage III), or fecal peritonitis (Hinchey stage IV) [15].

The physical status at admission was categorized into five groups using the American Society of Anesthesiologists ASA score [16].

Survival time was defined as time from first admission with acute colonic diverticulitis until death, regardless of cause. Patients who were alive at the end of April 2013 counted as cencored cases. The cause of death was available in the records of 613 patients, and for the remaining 37 patients, cause of death was obtained from the Cause of Death Registry, Statistics Norway.

Major endpoints, short- and long-term relative survival

The primary study endpoint was death. Short-term relative survival was reported as 100-day relative survival. Long-term relative survival was estimated for the patients who had survived the first 100 days to see if the patients who survived the acute episode of acute diverticulitis still had excess mortality compared to the general population. The long-term survival was calculated from the first admission for acute diverticulitis.

\section{Statistical methods}

Relative survival aims to measure mortality in excess of what would be expected for the study population if it did not have acute diverticulitis. In relative survival analysis, the observed survival in the group with diverticulitis is divided by the expected survival of a comparable group in the general Norwegian population with respect to age, sex, and calendar year of investigation. Using relative survival, consideration was made for the change in the age and gender in the population during the 25 years of study, and figures for excess mortality in the study population were given directly. Relative survival was estimated using the Ederer II method and analyzed with STATA 12 (Stata Corp LP, College Station, TX, USA) [17, 18]. Multivariable analyses were done using a full likelihood approach. Norwegian population survival probabilities for every year from 1988, by sex and age, were downloaded from the Human Mortality Database [19].

Two-sided $p$ values $<0.05$ were considered significant. Ninety-five percent confidence intervals (CIs) are reported where relevant. Medians are reported with range (minimum to maximum) where relevant.

\section{Results}

Study population and primary treatment

Baseline figures for the subcategories of diverticulitis in the 650 patients with 851 different admissions are given in Table 1. Diverticulitis with perforation leading to purulent or fecal peritonitis occurred in 56 patients. Eighty-nine percent $(50 / 56)$ of the perforations occurred during the patients' first admission. Among the 552 patients with uncomplicated diverticulitis at the first admission, 123 (22.3\%) were later readmitted, mostly with uncomplicated disease. After complicated diverticulitis, readmissions were less frequent. Patients with abscess formation had the longest time interval between onset of symptom until admission to hospital, 7 days (1-35).

A surgical procedure was performed in $88 / 650$ patients (13.5\%). A total of 80 were operated at their first admission, 4 at the second admission, 2 at their third admission, 1 at the forth admission, and 1 at the tenth admission. Hartmann procedure was performed in 54 patients, exploratory laparotomy in 13, resection with primary anastomosis and diverting stoma in 9, primary anastomosis without stoma in 7 , peritoneal lavage and drainage in 2, laparotomi with drainage of abscess in 2, and only proximal stoma in 1. Radiological abscess drainage was done in nine patients. Antibiotics had been given to all patients with complicated diverticulitis and in 726/738 admissions (98\%) with uncomplicated diverticulitis.

During the observation time, 219 patients were dead and 431 were still alive. The median observation time was 6.95 years $(0.28-24.66)$ or until death.

\section{Short-term survival}

The total 100-day mortality rate was $7.5 \%$ (49/650). Fortyone patients died during their first admission. Six patients died 
Table 1 Baseline characteristics in relation to subcategory of acute diverticulitis. Medians and ranges are given

\begin{tabular}{|c|c|c|c|c|c|}
\hline & \multicolumn{5}{|c|}{ Subcategory of acute diverticulitis during the first admissions in 650 patients } \\
\hline & $\begin{array}{l}\text { Uncomplicated } \\
(n=552)\end{array}$ & $\begin{array}{l}\text { Perforated with } \\
\text { abscess } \\
(n=38)\end{array}$ & $\begin{array}{l}\text { Perforated with } \\
\text { purulent peritonitis } \\
(n=42)\end{array}$ & $\begin{array}{l}\text { Perforated with } \\
\text { fecal peritonitis } \\
(n=8)\end{array}$ & $\begin{array}{l}\text { With acute intestinal } \\
\text { obstruction } \\
(n=10)\end{array}$ \\
\hline Age & $64(24-98)$ & $69(43-93)$ & $66(27-93)$ & $76(31-91)$ & $78(69-94)$ \\
\hline Male sex $(\%)$ & $229(41.5)$ & $14(36.8)$ & $17(40.5)$ & $2(25.0)$ & $3(30.0)$ \\
\hline Time interval $^{\mathrm{a}}$ & $2(0-30)$ & $7(1-21)$ & $2(1-30)$ & $1(1-5)$ & $4(1-14)$ \\
\hline \multicolumn{6}{|l|}{ CRP } \\
\hline At admission & $91(1-452)$ & $150(33-366)$ & $231(0-620)$ & $6(5-299)$ & $96(8-271)$ \\
\hline Highest & $114(1-453)$ & $223(33-524)$ & $301(26-620)$ & $273(5-417)$ & $119(59-363)$ \\
\hline Hospital stay in days & $3(0-30)$ & $7(1-35)$ & $10(0-73)$ & $13(1-31)$ & $14(7-22)$ \\
\hline \multicolumn{6}{|c|}{ Patients readmitted after first admissions } \\
\hline No & 429 & 34 & 39 & 8 & 9 \\
\hline Yes & 123 & 4 & 3 & 0 & 1 \\
\hline No. of readmissions & 191 & 5 & 4 & 0 & 1 \\
\hline \multicolumn{6}{|c|}{ Subcategory of diverticulitis during readmissions } \\
\hline Uncomplicated & 178 & 4 & 3 & 0 & 1 \\
\hline Abscess & 5 & 1 & & & \\
\hline Purulent peritonitis & 5 & & & & \\
\hline Fecal peritonitis & 1 & & & & \\
\hline With acute obstruction & 2 & & 1 & & \\
\hline
\end{tabular}

${ }^{\text {a }}$ Days from first symptom to admission

after a second admission and two after a third admission because of acute diverticulitis during this time interval. The 100-day relative survival curves in relation to the subcategories of acute diverticulitis are shown in Fig. 1.

Table 2 shows the results of unadjusted and adjusted analysis of relative survival during the first 100 days after the first admission for acute colonic diverticulitis. In the multivariable

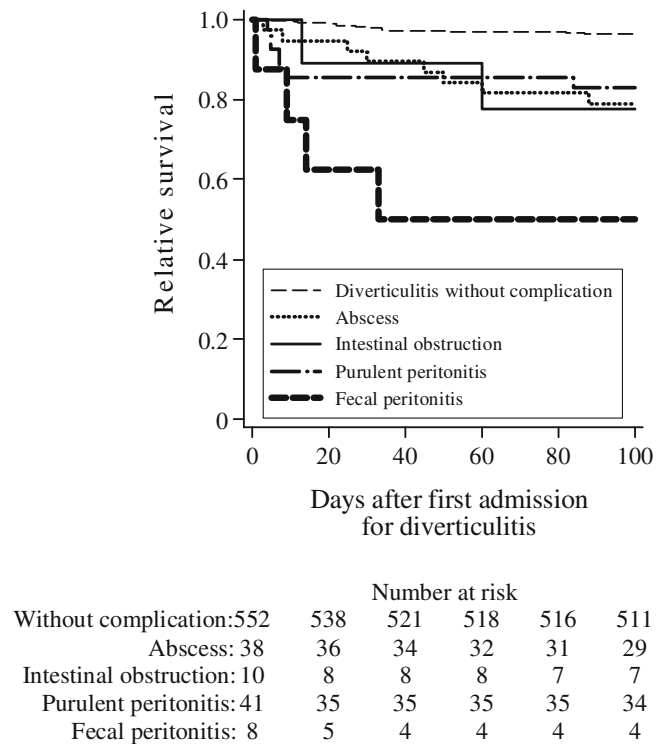

Fig. 1 Relative survival 100 days after first episode of acute colonic diverticulitis analysis, higher ages and high ASA scores, abscess formation, and perforation with fecal peritonitis were significantly associated with a shorter 100-day relative survival. Perforation with fecal peritonitis was the most serious form of the disease with high mortality and with an adjusted hazard ratio of 10.5 (CI 3.12 to 35.1) compared to uncomplicated acute colonic diverticulitis.

Diagnosis specific short-term mortality

Table 3 shows the main causes of a short-term lethal outcome. In patients with perforation, the main cause of death was septic complications (11/12). Ten out of 19 (53\%) patients with uncomplicated diverticulitis died of cardiovascular disease.

\section{Long-term survival}

Six hundred and nine patients survived the first 100 days after the first admission. In this group, the estimated relative survival decreased slightly by the years and was $96 \%$ (CI 92 to 100 ) after 5 years, $91 \%$ (CI 84 to 97 ) after 10 years, and $86 \%$ (CI 76 to 95 ) after 15 years. The corresponding figures after uncomplicated diverticulitis were $97 \%$ (CI 92 to 100) after 5 years, $91 \%$ (CI 84 to 98 ) after 10 years, and $87 \%$ (CI 76 to 97) after 15 years. After diverticulitis with abscess formation or free perforation (Hinchey I-IV), it was was $91 \%$ (CI 75 to 
Table 2 Multivariable analysis of 100 days relative survival after first admission for acute colonic diverticulitis

No. (\%) $N=650$ Unadjusted hazard ratio $p$ value 100-day relative survival Adjusted hazard ratio $p$ value

\begin{tabular}{|c|c|c|c|c|c|c|}
\hline \multicolumn{7}{|l|}{ Gender } \\
\hline Male & $265(40.8)$ & 1 & & $95 \%(92$ to 98$)$ & 1 & \\
\hline Female & $385(59.2)$ & $1.30(0.68$ to 2.51$)$ & 0.43 & $94 \%(91$ to 94$)$ & $0.71(0.35$ to 1.46$)$ & 0.35 \\
\hline \multicolumn{7}{|l|}{ Age } \\
\hline$<65$ years & $321(49.4)$ & 1 & & $100 \%(98$ to 100$)$ & 1 & \\
\hline $65-79$ years & $211(32.5)$ & 32.0 (4.2 to 242$)$ & 0.001 & $91 \%(86$ to 94$)$ & $15.9(2.03$ to 123$)$ & 0.008 \\
\hline $80+$ years & $118(18.2)$ & $60.4(7.9$ to 458$)$ & $<0.001$ & $86 \%(78$ to 92$)$ & $15.9(1.97$ to 129$)$ & 0.010 \\
\hline \multicolumn{7}{|l|}{ ASA score } \\
\hline $1-2$ & $461(70.9)$ & 1 & & $99 \%$ (98 to 100$)$ & 1 & \\
\hline 3 & $163(25.1)$ & 12.1 (4.8 to 31.0$)$ & $<0.001$ & $90 \%(85$ to 94$)$ & $4.5(1.7$ to 11.7$)$ & $<0.001$ \\
\hline 4 & $26(3.9)$ & $123(47.1$ to 320$)$ & $<0.001$ & $39 \%(22$ to 55$)$ & 37.1 (11.7 to 118$)$ & $<0.001$ \\
\hline \multicolumn{7}{|l|}{ Type of diverticulitis } \\
\hline Uncomplicated & $552(8.9)$ & 1 & & $97 \%(95$ to 99$)$ & 1 & \\
\hline Abscess formation & $38(5.8)$ & $7.0(3.05$ to 16.1$)$ & $<0.001$ & $79 \%(62$ to 89$)$ & $3.26(1.34$ to 7.93$)$ & 0.009 \\
\hline Perforation with purulent peritonitis & $42(6.5)$ & $5.4(2.3$ to 12.9$)$ & $<0.001$ & $84 \%(69$ to 92$)$ & $1.23(0.42$ to 3.53$)$ & 0.71 \\
\hline Fecal peritonitis & $8(1.2)$ & $30.4(10.3$ to 90$)$ & $<0.001$ & $44 \%(10$ to 74$)$ & $10.5(3.12$ to 35.1$)$ & $<0.001$ \\
\hline With intestinal obstruction & $10(1.5)$ & $7.1(1.6$ to 30.9$)$ & 0.009 & $80 \%(38$ to 96$)$ & $1.26(0.24$ to 6.51$)$ & 0.78 \\
\hline
\end{tabular}

${ }^{\text {a }}$ Adjusted for all factors

$100)$ after 5 years, $85 \%$ (CI 62 to 103 ) after 10 years, and $69 \%$ (CI 40 to 95) after 15 years. Figure 2 shows the estimated relative long-term survival in all patients in relation to ASA score at admission. All patients with ASA score 4 died within 3 years. Having survived the first 100 days, only one patient with acute diverticulitis later died directly after a new episode of acute diverticulitis. This episode was complicated with perforation, peritonitis, and sepsis.

Table 4 shows the results of unadjusted and adjusted analyses of different factors in relation to long-term relative survival in patients who survived the first 100 days after the first admission for acute colonic diverticulitis. The long-term survival beyond 100 days was unrelated to the different subtypes of acute diverticulitis, while higher ages, male gender, and higher ASA scores were associated with worse survival.

Diagnosis specific long-term mortality

Table 5 shows the main causes of death later than 100 days after the first admission of acute colonic diverticulitis. This is compared with the distribution of the main causes of death in North Trondelag county during 1988-2011. The proportion of patients who died from cardiovascular diseases, cancer, or bacterial infections was quite similar, while death caused by chronic obstructive pulmonary disease was more frequent in patients who had had diverticulitis.

Table 3 Causes of death during the first 100 days after the first admission for acute colonic diverticulitis, in relation to type of diverticulitis in 19882012

Main cause of death

Type of acute diverticulitis

\begin{tabular}{|c|c|c|c|c|}
\hline $\begin{array}{l}\text { Uncomplicated } \\
\text { diverticulitis } \\
(n=552)\end{array}$ & $\begin{array}{l}\text { With abscess } \\
(n=38)\end{array}$ & $\begin{array}{l}\text { Perforated with } \\
\text { purulent peritonitis } \\
(n=42)\end{array}$ & $\begin{array}{l}\text { Perforated with } \\
\text { fecal peritonitis } \\
(n=8)\end{array}$ & $\begin{array}{l}\text { With acute intestinal } \\
\text { obstruction } \\
(n=10)\end{array}$ \\
\hline
\end{tabular}

Cardiovascular disease

$10 \quad 2$

Cancer

2

2

Sepsis

2

Other

Total

19/552 (3.4 \%)

2

4

$8 / 38(21 \%)$
1

$4 / 8(50 \%)$
1

$1^{\mathrm{b}}$

$2 / 10(20 \%)$

${ }^{a}$ One each of fulminant hepatitis, uremia, rheumatoid arthritis with multi organ failure, Wernicke's encephalopathy, and one unknown

${ }^{\mathrm{b}}$ Femoral neck fracture 


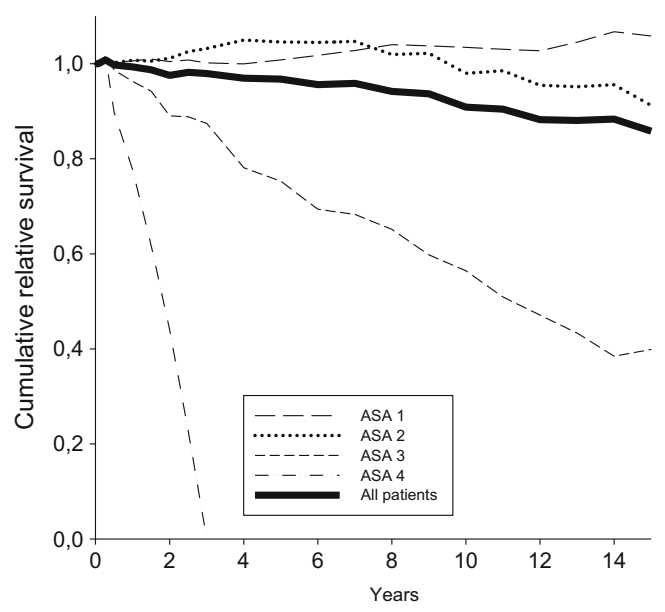

Fig. 2 Long-term relative survival in patients having survived beyond 100 days after first admission for acute diverticulitis, in relation to ASA score

\section{Discussion}

The present study confirmed high short-term mortality after perforated diverticulitis. In the group of patients who survived the first 100 days, the long-term survival was the same irrespective of type of diverticulitis and was only slightly curtailed compared to the Norwegian general population. During the entire study period, the patients were admitted from a defined geographical area that is served by one primary hospital which treats all categories of acute diverticulitis. Though the results may not be directly comparable with results from large referral centers because some patients with complicated diverticulitis might be referred to larger centers, it is worth considering that some patients with a grave prognosis may not be transportable and may die at the local hospital. Nonetheless, the present hospital most likely had a larger proportion of patients with uncomplicated disease compared to a center serving as a referral hospital.

The age distribution and death rates in the general population have changed during 25 years. In using relative survival analysis instead of overall survival analysis, we adjusted for these changes during the study period.

\section{Short-term survival}

Short-term survival is commonly reported as inhospital mortality or 30-day mortality. The present report chose 100-day mortality to denote short-term survival whether the patient died in hospital or not. Although most complications after a serious infection or an operation are evident within the first 4 weeks after an operation, death frequently occurs much later because of efficient intensive care units. Therefore, The Accordion Severity Grading System of surgical complications has proposed that the time horizon for postoperative deaths should be extended to 100 days [20]. Thus, in the present study, the short-term relative survival was defined as survival 100 days after the first admission due to an acute colonic diverticulitis and included patients who had been operated and those who had not operated.

Mortality in patients with uncomplicated diverticulitis was infrequently caused directly by this infection itself. The main

Table 4 Multivariable analysis of long term relative survival in patients who survived the first 100 days after the first admission for acute colonic diverticulitis

No. (\%) $N=609$ Unadjusted hazard ratio $p$ value 5-year relative survival Adjusted ${ }^{\mathrm{a}}$ hazard ratio $p$ value

\begin{tabular}{|c|c|c|c|c|c|c|}
\hline \multicolumn{7}{|l|}{ Gender } \\
\hline Male & $251(41.2)$ & 1 & & $95 \%(88$ to 100$)$ & 1 & \\
\hline Female & $358(58.8)$ & $0.93(0.63$ to 1.37$)$ & 0.70 & $98 \%$ (92 to 102$)$ & $0.66(0.46$ to 0.95$)$ & 0.023 \\
\hline \multicolumn{7}{|l|}{ Age } \\
\hline$<65$ years & $319(52.4)$ & 1 & & $99 \%$ (95 to 101$)$ & 1 & \\
\hline $65-79$ years & $192(31.5)$ & $4.43(2.75$ to 7.15$)$ & $<0.001$ & $97 \%$ (89 to 103$)$ & 4.22 (2.58 to 6.89$)$ & $<0.001$ \\
\hline $80+$ years & $98(16.1)$ & 15.5 (9.59 to 25.2$)$ & $<0.001$ & $86 \%$ (64 to 107$)$ & $9.80(5.65$ to 17.0$)$ & $<0.001$ \\
\hline \multicolumn{7}{|l|}{ ASA-score } \\
\hline $1-2$ & 455 (74.7) & 1 & & $104 \%$ (100 to 107$)$ & 1 & \\
\hline 3 & $145(23.8)$ & $5.90(4.03$ to 8.64$)$ & $<0.001$ & $76 \%(63$ to 87$)$ & 2.51 (1.69 to 3.72 ) & $<0.001$ \\
\hline 4 & $9(1.5)$ & $30.9(13.0$ to 73.3$)$ & $<0.001$ & $0 \%(0$ to 0$)$ & 7.74 (2.92 to 20.5$)$ & $<0.001$ \\
\hline \multicolumn{7}{|l|}{ Type of diverticulitis } \\
\hline Uncomplicated & $533(87.5)$ & 1 & & $97 \%(92$ to 100$)$ & 1 & \\
\hline Abscess formation & $29(4.8)$ & $1.22(0.50$ to 2.98$)$ & 0.67 & $98 \%$ (70 to 112$)$ & $1.11(0.51$ to 2.40$)$ & 0.79 \\
\hline Perforation with purulent peritonitis & $35(5.8)$ & $1.26(0.62$ to 2.58$)$ & 0.52 & $85 \%(65$ to 97$)$ & $1.26(0.62$ to 2.56$)$ & 0.52 \\
\hline Fecal peritonitis & $4(0.7)$ & $2.4 \times 10^{-6}(0)$ & 0.99 & $107 \%(107$ to 107$)$ & $3.0 \times 10^{-6}$ & 0.99 \\
\hline With intestinal obstruction & $8(1.3)$ & $2.28(0.71$ to 7.28$)$ & 0.16 & $137 \%$ (53 to 156$)$ & $1.21(0.39$ to 3.72$)$ & 0.74 \\
\hline
\end{tabular}

${ }^{a}$ Adjusted for all factors 
Table 5 Distribution of causes of death of 178 patients who died after the first 100 days after the first admission for acute colonic diverticulitis, compared with the causes of death in North Trondelag county during 1988-2011

\begin{tabular}{lll}
\hline Main cause of death & $\begin{array}{l}\text { Patient dead more than } 100 \text { days after acute diverticulitis } \\
n=178\end{array}$ & $\begin{array}{l}\text { Total deaths in North Trondelag county } \\
n=29,449\end{array}$ \\
\hline Cardiovascular disease & $75(42 \%, 33$ to 53$)$ & $13,037(44.3 \%, 43.5$ to 45.0$)$ \\
Cancer, all forms & $35(20 \%, 14$ to 27$)$ & $6,681(22.7 \%, 22.1$ to 23.2$)$ \\
Bacterial infections, including pneumonia & $17(10 \%, 5.6$ to 15$)$ & $1,734(5.9 \%, 5.6$ to 6.2$)$ \\
Chronic obstructive pulmonary disease & $18(10 \%, 6.0$ to 16$)$ & $1,239(4.2 \%, 4.0$ to 4.4$)$ \\
Other & $26(15 \%, 9.5$ to 21$)$ & $6,758(22.9 \%, 22.4$ to 23.5$)$ \\
Unknown & $7(4 \%, 1.6$ to 8.1$)$ & \\
\hline
\end{tabular}

Poisson exact $95 \%$ confidence intervals

causes were associated diseases, formost cardiovascular diseases, and an end stage of other serious diseases. Acute perforation with fecal peritonitis was the most serious complication, with a higher mortality rate than in cases with acute perforation and purulent peritonitis. In the multivariable analysis, patients with purulent peritonitis did not have a significantly reduced survival. This was probably a type II error. Haglund et al. in 1979 reported $24 \%$ mortality in 42 patients with diffuse peritonitis and $45 \%$ mortality in 7 patients with diffuse fecal peritonitis [21]. This was in accordance with findings in the present study as well as those of a recent Dutch study [14] that reported inhospital mortality in patients operated for all types of perforation as $26 \%$, highest in fecal peritonitis with $48 \%$. A British population-based study of patients with perforated diverticulitis found no mortality in Hinchey grade I, $17 \%$ in Hinchey grade II, $23 \%$ in Hinchey grade III, and $45 \%$ in Hinchey grade IV [11].

A study from a Finnish referral center reported an overall inhospital mortality of $2.3 \%$, being $1 \%$ in uncomplicated diverticulitis and only $5.5 \%$ among pasients admitted for diverticular perforations [4]. The low mortality may be related to selection bias compared to the present study. A nationwide Canadian study of prognosis in diverticulitis reported an inhospital mortality rate of $0.4 \%$ in the ages $51-60$ years and $3.3 \%$ above 60 years [6]. In some patients, death in the course of acute colonic diverticulitis was the end stage of another fatal disease, like cancer. In uncomplicated diverticulitis, cardiovascular disease was the main cause of death within 100 days. In the patients with a fatal short-term outcome after complicated diverticultis, septic complications predominated.

\section{Long-term survival}

The estimated long-term relative survival in patients who survived the first 100 days after acute diverticulitis was unrelated to the subtype of diverticulitis. All had a slightly reduced estimated relative survival, and this was related to accompanying diseases, as measured by the ASA score. Only one patient later died because of diverticulitis, this time with perforation. The rest died from other diseases. Similar findings were reported in a recent study [14].

The distribution of causes of death later than 100 days after the first admission in the study population was quite similar to the population of North Trondelag county during the same period.

One exception was chronic obstructive pulmonary disease, which was more prevalent in patients who had been hospitalized because of acute colonic diverticulitis. The relationship has not been reported before and may be of little importance. It could be related to a common pathogenic factor, like smoking [22] or soft tissue weakening, in both diverticulosis and chronic obstructive pulmonary disease.

Nearly all perforations happened during the first stay, and almost all deaths directly related to diverticulitis occurred during the first admission. The present study, therefore, supports the view held by others that elective resection for diverticulitis is not indicated when the intent is to reduce mortality caused by future episodes of diverticulitis. However, it may be considered if the goal is to improve the quality of life in ablebodied patients with continued severe symptoms, including stenosis or fistula [4, 9, 13, 21, 23, 24].

Weaknesses and limitations of the study

The study was retrospective in nature. We did not base the study only upon diagnosis lists, but controlled every patient's record for evidence of acute colonic diverticulitis. Nevertheless, the diagnosis would be even more reliable in a prospective study, which requires a set of diagnostic tests to confirm or disprove the diagnosis of acute colonic diverticulitis. The study included only patients treated in hospital. The findings might not be valid for patients with mild disease treated at home.

\section{Strengths}

The hospital served the whole population within a geographical area and treated the whole spectrum of hospitalized 
patients with acute colonic diverticulitis, including all complications. The endpoint, death, was a very robust parameter and known in all patients. We used relative survival analysis, instead of overall survival analysis. This made it possible to compare survival in the patients directly with survival in the general population and over as long period as 25 years.

\section{Conclusions}

The 100 days' relative survival was significantly reduced in perforated disease, particularly after fecal perforation. Even patients with uncomplicated diverticulitis had a small reduction of 100 days' survival, which was mainly due to accompanying diseases. In the patients who survived the first 100 days, the long-term survival was only slightly reduced. It was independent of the type of diverticulitis, but dependent on accompanying diseases.

Open Access This article is distributed under the terms of the Creative Commons Attribution License which permits any use, distribution, and reproduction in any medium, provided the original author(s) and the source are credited.

\section{References}

1. John SK, Teo NB, Forster AL (2007) A prospective study of acute admissions in a surgical unit due to diverticular disease. Dig Surg 24(3):186-190

2. Frileux P, Dubrez J, Burdy G, Roullet-Audy JC, Dalban-Sillas B, Bonnaventure F, Frileux MA (2010) Sigmoid diverticulitis. Longitudinal analysis of 222 patients with a minimal follow up of 5 years. Colorectal Dis 12(7):674-680

3. Hall JF, Roberts PL, Ricciardi R, Read T, Scheirey C, Wald C, Marcello PW, Schoetz DJ (2011) Long-term follow-up after an initial episode of diverticulitis: what are the predictors of recurrence? Dis Colon Rectum 54(3):283-288

4. Makela JT, Kiviniemi HO, Laitinen ST (2010) Spectrum of disease and outcome among patients with acute diverticulitis. Dig Surg 27(3):190-196

5. Tursi A, Brandimarte G, Giorgetti G, Elisei W, Maiorano M, Aiello F (2008) The clinical picture of uncomplicated versus complicated diverticulitis of the colon. Dig Dis Sci 53(9):2474-2479

6. Razik RA, Chong CA, Nguyen GC (2013) Younger age and prognosis in diverticulitis: A nationwide retrospective cohort study. Can J Gastroenterol 27(2):95-98
7. Ritz JP, Lehmann KS, Frericks B, Stroux A, Buhr HJ, Holmer C (2011) Outcome of patients with acute sigmoid diverticulitis: multivariate analysis of risk factors for free perforation. Surgery 149(5): 606-613

8. Hansen O, Graupe F, Stock W (1998) Early elective surgery of acute uncomplicated sigmoid diverticulitis - a dangerous mistake? Chirurg 69(4):443-449

9. Chapman J, Davies M, Wolff B, Dozois E, Tessier D, Harrington J, Larson D (2005) Complicated diverticulitis: is it time to rethink the rules? Ann Surg 242(4):576-581

10. Constantinides VA, Tekkis PP, Senapati A (2006) Prospective multicentre evaluation of adverse outcomes following treatment for complicated diverticular disease. Br J Surg 93(12):1503-1513

11. Morris CR, Harvey IM, Stebbings WS, Hart AR (2008) Incidence of perforated diverticulitis and risk factors for death in a UK population. Br J Surg 95(7):876-881

12. Humes DJ, West J (2012) Role of acute diverticulitis in the development of complicated colonic diverticular disease and 1-year mortality after diagnosis in the UK: population-based cohort study. Gut 61(1): $95-100$

13. Binda GA, Arezzo A, Serventi A, Bonelli L (2012) Multicentre observational study of the natural history of left-sided acute diverticulitis. Br J Surg 99(5):276-285

14. Vermeulen J, Gosselink MP, Hop WC, van der Harst E, Hansen BE, Mannaerts GH, Coene PP, Weidema WF, Lange JF (2011) Long-term survival after perforated diverticulitis. Colorectal Dis 13(2):203209

15. Hinchey EJ, Schaal PG, Richards GK (1978) Treatment of perforated diverticular disease of the colon. Adv Surg 12:85-109

16. American Society of Anesthesiologists ASA score (2013). http:// www.asahq.org/

17. Dickman PW, Sloggett A, Hills M, Hakulinen T (2004) Regression models for relative survival. Stat Med 23(1):51-64

18. Dickman PW and Coviello EMHM (2013) Estimating and modelling relative survival. In Estimating and modelling relative survival. http:// pauldickman.com/survival/strs.pdf.

19. Human Mortality Database (2013) Human mortality database. In http://www.mortality.org/.

20. Strasberg SM, Linehan DC, Hawkins WG (2009) The accordion severity grading system of surgical complications. Ann Surg 250(2):177-186

21. Haglund U, Hellberg R, Johnsen C, Hulten L (1979) Complicated diverticular disease of the sigmoid colon. An analysis of short and long term outcome in 392 patients. Ann Chir Gynaecol 68(2):41-46

22. Hjern F, Wolk A, Hakansson N (2011) Smoking and the risk of diverticular disease in women. Br J Surg 98(7):997-1002

23. van de Wall BJ, Draaisma WA, van Iersel JJ, Consten EC, Wiezer MJ, Broeders IA (2013) Elective resection for ongoing diverticular disease significantly improves quality of life. Dig Surg 30(3):190 197

24. Van Arendonk KJ, Tymitz KM, Gearhart SL, Stem M, Lidor AO (2013) Outcomes and costs of elective surgery for diverti.cular disease: a comparison with other diseases requiring colectomy. JAMA Surg 148(4):316-321 